\title{
PERSPECTIVE
}

\section{The management of cytomegalovirus retinitis in AIDS}

\author{
Baljean Dhillon
}

Cytomegalovirus (CMV) shares many properties with other members of the herpesvirus family - it exhibits latency, reactivates under suitable conditions to cause human disease, and it is incurable. CMV is the primary opportunistic viral infection in the immunosuppressed person and gives rise to a wide spectrum of disease. CMV is a major cause of morbidity and mortality among patients with AIDS, and the retina is the commonest site of infection. ${ }^{1}$ Cytomegalovirus retinitis (CMVR) may be the AIDS defining diagnosis though more commonly occurs months after the diagnosis of AIDS. If left untreated, patients with unilateral CMVR are likely to develop disease in their second eye and ultimately become blind. ${ }^{2}$ It has now become accepted practice to treat sight threatening CMVR with either ganciclovir or foscarnet, which are effective in delaying the progress of this destructive infection. Systemic treatment for CMV disease also reduces extraretinal CMV related morbidity and mortality and subjectively improves quality of life. ${ }^{3}$ This review of the clinical management of CMVR in patients with AIDS also includes complications of therapy, and dilemmas which face the patient, physician, and ophthalmologist.

The use of antiretroviral agents together with the early recognition and treatment of opportunistic infection has led to the increased survival of patients with AIDS despite their profound immunodepletion. The median survival of patients with AIDS after the diagnosis of CMV disease is 1 year or longer, ${ }^{14}$ and, as with other evolving statistics concerning CMVR, this is likely to underestimate the current clinical experience. ${ }^{5}$ Given the increasing number of patients with HIV infection, and their longer survival, it is likely that CMVR will become an increasingly prevalent condition. A previous communication reported the increasing CMVR attack rate with prolonged survival, ${ }^{6}$ and supports the impression that the majority of patients with AIDS would develop CMVR if they survived long enough.

Reports from the United States of CMVR prevalence of up to $40 \%$ among patients with AIDS $^{2378}$ vary widely due to differences in patient recruitment, detection method, and populations studied. Geographical variations in survival which relate to the availability and use of drugs to treat opportunistic infections would also influence the prevalence of CMVR. Studies carried out prospectively or post mortem would yield the most accurate prevalence data. Regional series from London and Edinburgh estimated the minimum risk of developing sight threatening CMVR among patients with AIDS as $17 \%,{ }^{19}$ despite differences between the two populations in terms of HIV transmission group. In Edinburgh $38 \%$ of AIDS is associated with homosexual/ bisexual behaviour, though this percentage constituted $75 \%$ of patients with CMVR in this region; this is probably a reflection of the higher prevalence of sexually acquired CMV infection. It could also represent greater immunosuppression in the homo/bisexual group because of the longer duration of infection. ${ }^{6}$ In this region CMVR is the index diagnosis in approximately $3 \%$ of patients with AIDS and $17 \%$ of patients with CMVR and AIDS.

\section{Symptoms}

In comparison with the more severe systemic symptoms associated with AIDS the onset of CMVR is associated with few visual symptoms; unless the patient is aware of their significance, relatively minor visual symptoms may be considered too trivial to disclose to his physician. In Edinburgh from a cohort of 24 patients with CMVR and AIDS, 16 had unilateral retinitis at presentation and, although $59 \%$ of this group had macula and/or optic nerve threatening disease, less than half the group showed visual symptoms. ${ }^{9}$ Visual blurring, photopsia, floaters, and scotomata are usually only noticed on occlusion of the unaffected eye, or when CMVR affects both eyes. Although untreated CMVR is relentlessly progressive, the spread is relatively slow, and may partly explain the absence of acute visual symptoms. The size and location of CMVR influence the rate of progression. ${ }^{10}$ The insidious and silently progressive nature of CMVR may result in advanced disease before symptoms become apparent, resulting in delayed diagnosis and treatment.

\section{Signs}

Patients with established CMVR may show mild vitreous activity and anterior chamber activity with endothelial deposits, though posterior synechiae formation is not observed. Although CMV produces a spectrum of fundus appearances, the clinical picture usually allows a diagnosis to be made without the need for viral isolation from the eye. However, uncertainty may arise - early lesions of CMVR in evolution may mimic the cotton wool spots of HIV retinopathy. Weekly follow up will confirm the diagnosis of CMVR if the signs of enlargement, retinal oedema and necrosis, and vasculitis are observed. In the early stages the retina shows white granular patches with irregular margins and variable overlying haemorrhage. CMVR tends to spread along one or more of the vascular arcades (most commonly the temporal branches), resulting in wedge shaped areas of necrosis with the apices pointing posteriorly. Vascular sheathing may be subtle, or florid enough to appear as frosted branch angiitis." CMVR may present as extensive, oedematous lesions which progress more rapidly than the indolent granular lesion. ${ }^{10}$ Progression occurs by centrifugal or 'brushfire' spread from the original focus. The advancing edge consists of oedematous, opaque retina and this border may show faint grey/white 'satellite' retinal opacities. The resulting central atrophic scar shows variable pigment epithelial disturbance with areas of retinal gliosis, which appears densely white and may even show intraretinal calcific changes. ${ }^{12}$ Retinal vasculature within the affected area shows attenuation and eventually becomes 'ghost' vessels. CMVR produces a full thickness retinal necrosis and can lead to rhegmatogenous retinal detachment. This is more likely to occur with CMVR affecting a large peripheral area of retina and in these patients the cumulative probability of $50 \%$ detachment at 1 year after the diagnosis of CMVR. ${ }^{13}$ Small centrally located CMVR does not carry such a high risk of retinal detachment. 


\section{Evaluation}

Close observation and accurate documentation of the retinal signs are the key to effective management of CMVR. The patients are often sick and unable to tolerate prolonged examination. Following assessment of the best corrected visual acuity, the pupils are dilated for fundus examination. The most important evaluation procedure is binocular indirect ophthalmoscopy. If a central focus of CMVR shows features of questionable activity and if the patient is able to sit at a slit-lamp, a hand held 78 dioptre or 90 dioptre lens is used to examine the fundi, which may then be photographed. Perimetry can be of use though few patients are able to perform it well. Correlation of the retinal appearance with a corresponding field defect supports the diagnosis of CMVR; this invariably produces an absolute scotoma which enlarges as the infection progresses. ${ }^{14}$ When photography is not possible, perimetry can be attempted. However, both photography and perimetry are of little value in mapping the progression of peripheral retinitis anterior to the equator, which is inaccessible to the fundus camera and may cause no scotoma. Also, it is not practical to perform photography or perimetry in patients with advanced AIDS who require bedside examination, and at this stage there is a high risk of recurrence. Therefore, it is always necessary to document not only the characteristics of the retinitis, as described above, but also precisely to record the site and extent of the CMVR by clear and accurate drawing. Holland $e t a l^{15}$ described a classification for recording the site which divides the retina into three zones - a posterior area which encompasses the disc and macula (zone 1), extending $3000 \mu \mathrm{m}$ from the fovea and $1500 \mu \mathrm{m}$ from the disc; the midperipheral retina (zone 2) extending anterior to zone 1 up to the equator; and the far peripheral retina (zone 3) extending anterior to the equator. For peripheral CMVR the site is further defined by documenting the retinal site and clock hours involved; retinal vessels and vortex vein ampullae adjacent to the infection border served as landmarks to identify the demarcation line between affected and unaffected retina. The vascular landmarks adjacent to the CMVR border are noted, using the smallest visible arteriolar and venular branches to describe the boundary position, particularly the border closest to the macula and optic nerve.

\section{Fundus fluorescein angiography}

This investigation is not necessary for the majority of patients. It may be helpful in distinguishing between CMVR and toxoplasmic retinochoroiditis if the clinical picture is uncertain. The fluorescein angiogram appearance of CMVR shows evidence of retinal vascular occlusion and permeability alterations in the area of retinitis. ${ }^{16}$ Hyperfluorescence starts at the centre, and extends to the borders, and the final hyperfluorescent area is smaller than the lesion observed on the red free photographs. ${ }^{17}$ The underlying choroid is still present, and alterations in the retinal pigment epithelium produce this angiographic picture.

Toxoplasmic retinochoroiditis produces complete destruction of the retina and choroid, and angiography shows marked fluorescein staining without permeability changes or artery obstruction in the areas of focal periarterial exudate or plaques ${ }^{16}$; inflammatory changes in the vitreous and anterior chamber ${ }^{18}$ obscure the hyperfluorescence, which starts at the edge of the lesion and progresses towards the centre. The area of late hyperfluorescence is larger than the lesion seen on the control photographs. ${ }^{17}$

\section{Laboratory investigations}

Serological testing or isolation of CMV from the throat or urine are not required for making the diagnosis of CMVR, which is a sign of systemic infection. Of 24 patients with AIDS and CMVR studied in Edinburgh, 19 had raised CMV IgG levels indicating past infection, nine of whom also had detectable CMV IgM indicating newly acquired infection; $95 \%$ of homosexual men and $80 \%$ of HIV positive injecting drug users in Edinburgh show evidence of past CMV infection (Regional Virus Laboratory, unpublished results).

The development of in situ nucleic acid hybridisation and nucleic acid amplification by polymerase chain reactions will allow laboratory diagnosis on ocular fluids and tissue. ${ }^{19} 20$ Until these techniques are widely available ophthalmologists must rely on their clinical skills to distinguish between CMVR and other infections affecting the retina and/or the choroid, caused by toxoplasmosis, syphilis, candida, pneumocystis, varicella zoster, and herpes simplex, which may cause concurrent infection. This article does not intend to cover the wide spectrum of differential diagnoses, though the reader should become familiar with their distinguishing clinical features.

\section{Treatment}

In most instances the treatment for CMVR is for the duration of the patient's life and decisions regarding therapy should actively involve the patient and his partner. Even if CMVR is peripheral and not immediately sight threatening, treatment should not be delayed; CMVR threatening the macula and disc should be treated immediately. A heavy commitment is required from the patient, ophthalmologist, and referring physician to monitor the therapeutic response and toxic effects of therapy for the duration of the patient's life. The problem of progressive CMVR during maintenance therapy remains a major management challenge.

\section{Systemic therapy}

A controlled retrospective study of ganciclovir ${ }^{15}$ confirmed previous uncontrolled reports of treatment benefit for CMVR. Foscarnet is also effective in slowing the progression of CMVR. ${ }^{21}$ Intravenous ganciclovir and foscarnet suppress, but do not eradicate CMV disease. Following high dose induction therapy for 2-3 weeks, long term maintenance therapy is necessary to prevent recurrence of CMVR, which nevertheless occurs in $18-54 \%$ of patients. ${ }^{22-24}$ It is likely that all patients will relapse if the survival is sufficiently long ${ }^{25}$ though this generally responds to an increase in anti-CMV therapy. Both drugs are associated with toxic side effects, ganciclovir with bone marrow suppression and foscarnet with renal impairment, metabolic abnormalities, and seizure. Foscarnet therapy is associated with an increased survival period ${ }^{4}$; however, patients with impaired renal function had a longer survival with ganciclovir. Overall ganciclovir was better tolerated than foscarnet which requires a prolonged infusion time and the maintenance of adequate hydration is often poorly tolerated.

Partial or complete arrest of active CMVR occurs in almost all patients after induction therapy with ganciclovir at a dose of $10 \mathrm{mg} / \mathrm{kg} /$ day (two divided doses), or $180 \mathrm{mg} / \mathrm{kg} /$ day (three divided doses) of foscarnet. ${ }^{34152123}$ Maintenance regimens are daily infusions of ganciclovir $5.0 \mathrm{mg} / \mathrm{kg}$ daily or foscarnet 90 $\mathrm{mg} / \mathrm{kg}$ for 5-7 days per week. Induction treatment for CMVR is ideally administered in a hospital or hospice setting, and maintenance therapy can be carried out at home with the assistance of the general practitioner, district nurse, partner, or friend. A central line facilitates maintenance treatment, though generalised septicaemia caused by indwelling central venous lines in patients with AIDS is a life threatening risk. ${ }^{26}$ The use of positive pressure reservoirs for intravenous therapy - for example, the portable Intermate infusion delivery system, simplifies home maintenance therapy and 
allows the patient greater mobility during the infusions. Nursing and medical support in the community are necessary for effective domiciliary treatment, otherwise problems such as central line infections and poor compliance may pass unnoticed. In view of the practical difficulties in achieving adequate hydration with foscarnet, ganciclovir is still preferred for maintenance therapy. In vitro studies raise the possibility of synergism ${ }^{27}$ between the two antivirals, and in future both ganciclovir and foscarnet may be used in an alternating regimen; this may have the added advantage of preventing the development of CMV resistance. ${ }^{28}$

\section{Local therapy}

There is great interest in local therapy for CMVR particularly for patients who have problems with standard treatment regimens and in countries where resources are restricted. Intravitreal ganciclovir has been reported to be effective in controlling CMVR in patients who cannot tolerate systemic therapy ${ }^{29-32}$; this requires twice weekly injections of $200 \mu \mathrm{g}$ of ganciclovir in $0.1 \mathrm{ml}$ sterile water for $2-3$ weeks, and weekly intravitreal injections are used as maintenance treatment. An alternative regimen has been described using intravitreal foscarnet $1200 \mu \mathrm{g}$ in $0.05 \mathrm{ml}$ of sterile filtered solution though there are limited data on its efficacy. ${ }^{33}$ Sustained release implants delivering intraocular ganciclovir have been developed with a duration of delivery of approximately 4 months. Early reports are encouraging, ${ }^{34}$ though there are risks of vitreous haemorrhage, retinal detachment, malposition, and infection. Randomised, controlled trials against standard therapy are necessary to define their place in the management of CMVR. ${ }^{25}$

Other forms of local treatment include transscleral iontophoresis of foscarnet ${ }^{35}$ and laser photocoagulation to produce a barrier $\mathrm{scar}^{36}$; these treatment modalities require further study but are exciting possibilities for the future.

\section{Treatment toxicity}

In most UK centres ganciclovir remains the first line therapy, though toxicity is a common problem. Ganciclovir associated neutropenia is often associated with concomitant zidovudine administration; the use of alternative antiretroviral agents for example, $2^{\prime}, 3^{\prime}$-dideoxyinosine (ddi) - may lead to a reduction in this side effect. Ganciclovir associated myelotoxicity should be managed by the following strategies:

- withholding zidovudine

- using haematological stimulating factors (G-CSF)

- replacing ganciclovir with foscarnet

- using a combination of foscarnet and ganciclovir

- intravitreal therapy

\section{Response to therapy}

Following the initiation of treatment for CMVR, ophthalmoscopic monitoring of both eyes is necessary and the guidelines described below for the timing of ophthalmic examination are based on local experience. Changes in the appearance of the border infection usually accompany a response to induction therapy, and patients should be examined weekly during this period. After the first week of treatment signs of active CMVR may still be present with oedematous, opaque, and variably haemorrhagic retina at the infected boundary. By the second or third week of induction a healing response is seen with decreased opacification at the infected border and a more granular appearance to the retina with resolution of the satellite spots; most importantly, failure of the borders to advance signifies infection control. Cessation in centrifugal spread of CMVR is the major criterion used to assess a therapeutic response as altered border characteristics may not be a reliable sign of non-progression. Thereafter, the frequency of follow up is determined by factors such as the site, laterality, control of infection (cessation of CMVR spread), and the appearance of the CMVR border. If the CMVR border is close to and threatening the macula/optic nerve, with signs of border activity and advancement, frequent follow up every 1-2 weeks is necessary. If there is no sign of progression, and the border looks inactive follow up might be lengthened to 4-6 weekly; patients with bilateral CMVR are more likely to relapse during maintenance therapy ${ }^{9}$ and should be followed more frequently. A reduction in the frequency of maintenance treatment from 7 to 5 days per week should only be considered for patients with stable and inactive CMVR. Further reduction is likely to lead to relapse. A diagnosis of relapsing or breakthrough CMVR is made by serial examinations showing sequential creeping into previously unaffected retina, and is an indication for increased treatment. In the event of breakthrough infection, increasing the frequency of treatment from 5 to 7 days per week may be sufficient to achieve control. If progression continues, repeat induction is indicated. 'Smouldering' breakthrough CMVR must be distinguished from persistent white border opacification which does not advance, and is likely to represent atypical healing rather than active infection. ${ }^{12}$ Failure to make this distinction may lead to unnerecsary alterations in treatment.

\section{Prophylaxis for the fellow eye}

Without therapy the likelihood of CMVR in the second eye of patients presenting with unilateral disease is estimated to be $60 \%$; however, with intravenous anti-CMV treatment this falls to $0-15 \%{ }^{22-24}$ Daily maintenance therapy places major restrictions on the patient's mobility and carries the likelihood of toxic side effects. If all useful vision in one eye is lost, and the other is unaffected by CMVR, this raises the dilemma concerning prophylaxis for the unaffected eye. The patient may continue maintenance treatment, though in the absence of a prospective study confirming the superior efficacy of - daily maintenance therapy in preventing involvement of the second eye, it is tempting to spare the patient therapy for 2 or more days per week. An alternative is to simply observe the patient at 4-weekly intervals and start treatment as soon as CMVR is detected. Holland $e t a l^{22}$ reported three patients who had no prophylaxis for the uninvolved eye and who remained free of CMVR followed up to 35 weeks. Withholding systemic prophylaxis may lead to extraretinal CMV disease occurring in the gastrointestinal tract, adrenal glands, central nervous system, or lungs: $16-38 \%$ of patients receiving intravitreal ganciclovir and no systemic prophylaxis develop extraocular disease. ${ }^{37}{ }^{38}$ Disseminated CMV infection is a cause of death in patients with AIDS and postmortem studies reveal occult CMV infection in most patients. ${ }^{39}$ The need for prophylaxis requires investigation by a randomised, controlled clinical trial and it may be appropriate to use oral ganciclovir $^{40}$ in this situation.

\section{Retinal detachment}

The surgical treatment for retinal detachment in patients with CMVR has been described ${ }^{5134-44}$ and vitrectomy and silicone oil tamponade, with or without scleral buckling, is successful in achieving anatomical success. The functional results of surgery in terms of visual acuity improvement are relatively poor owing to optic nerve disease, recurrent detachment, and progressive CMVR; therefore patient selection is important. The state of the fellow eye and the prognosis of the patient should be considered before surgery is undertaken - the stress of surgery may lead to a shortened life expectancy in the debilitated patient. Criteria for surgery 
include functional vision in the involved eye immediately before detachment, progressive detachment with macula involvement, extramacular detachment if the fellow eye has no useful vision, and a patient who is willing and able to withstand the rigours of surgery. More conservative modes of treatment - for example, laser photocoagulation - have been used to stop advancing non-bullous detachment, though appear ineffective in walling advancing CMVR.

\section{Surveillance and screening}

CMVR is commonly asymptomatic and a prospective study showed CMVR affected only the peripheral retina in $51 \%$ of patients with AIDS. ${ }^{3}$ Peripheral CMVR is not likely to be diagnosed until the disease advances into the posterior pole, where the physician may detect it using direct ophthalmoscopy, or a fall in the visual acuity occurs. The degree of retinal scarring and visual morbidity is reduced if CMVR is detected and treated early. In areas of the UK with high rates of HIV infection, ophthalmologists and physicians specialising in infectious diseases should be encouraged to develop local surveillance methods for detecting CMVR in order to prevent blindness among AIDS patients. At each routine visit the attending physician should inquire about visual symptoms. CMVR affecting the posterior retina may be asymptomatic, therefore ophthalmoscopy, preferably after pupil dilatation, should be part of any examination protocol. The indications for ophthalmic referral include patients with visual symptoms, the presence of suspicious retinal lesions, a rapidly CD4 lymphocyte count, and evidence of extraretinal CMV disease. The findings of a recent study ${ }^{45}$ support screening when the CD4 cell count falls to less than $50 \times 10^{6} / 1$ and this criterion is followed by many UK centres. The identification of other risk factors awaits further prospective studies.

Despite every effort to preserve vision and quality of life, progressive bilateral visual loss and increasing disability may occur. Although the survival outlook at this stage is poor, one should consider partially sighted or blind registration. Counselling, home assessment, and even occupational therapy services can be especially helpful for these relatively young, sick patients and their families, and registration should be expedited. If all useful vision is lost in both eyes or when the patient enters the terminal phase of his illness, treatment for CMVR should be withdrawn.

A major fear shared by patients with AIDS is the threat of blindness in their final months of life. CMVR is the leading cause of visual loss and the goal of management is to suppress infection and minimise treatment associated morbidity. This goal can only be achieved by the concerted efforts of the ophthalmologist working closely with other members of the AIDS health care team.

Princess Alexandra Eye Pavilion, Chalmers Street

BALJEAN DHILLON Edinburgh EH3 $9 H A$

1 Peters BS, Beck EJ, Anderson S, Coleman D, Coker R, Main J, et al. Cytomegalovirus infection in AIDS. Patterns of disease, response to therapy and trends in survival. $\mathcal{F}$ Infect 1991; 23: 123-8

2 Jabs DA, Enger C, Bartlett JG. Cytomegalovirus retinitis and acquired immunodeficiency syndrome. Arch Ophthalmol 1989; 107: 75-80.

3 Henderly DE, Freeman WR, Causey DM, Rao NA. Cytomegalovirus retinitis and response to therapy with ganciclovir. Ophthalmology 1987; 94: 425-32.

4 Studies of ocular complications of AIDS research group in collaboration with the AIDS clinical trials group. Mortality in patients with the acquired cytomegalovirus retinitis. N Engl f Med 1992; 326: 213-20.

5 Chuang EL, Davis JL. Management of retinal detachment associated with CMV retinitis in AIDS patients. Eye 1992; 6: 28-34.

6 Cheong I, Flegg PJ, Brettle RP, Welsby PD, Burns SM, Dhillon B, et al. CMV disease in AIDS: the Edinburgh experience. Int $\mathcal{Y}$ STD and AIDS 1992; 3 : 324-8.

7 Palestine AG, Rodrigues MM, Macher AM, Chan CC, Lane HC, Fauci AS Ophthalmic involvement in acquired immunodeficiency syndrome. Ophthalmology 1984; 91 : 1092-9.

8 Khadem M, Kalish SB, Goldsmith JA, Fetkenhour C, O'Grady RB, Phair JP.
Ophthalmologic findings in acquired immunodeticiency syndrome. Arch Ophthalmol 1984; 102: 201-4.

9 Dhillon B, Eddyshaw D, McClean H, Cheong I, Flegg P, Brettle R. Cytomegalo virus retinitis and AIDS in Edinburgh: presentation, therapy and visual outcome. Int $₹$ STD and AIDS 1993;4:339-41.

10 Holland GN, Sculer JD. Progression rates of cytomegalovirus retinopathy in ganciclovir-treated patients and untreated patients. Arch Ophthalmol 1992; 110: $1435-42$.

11 Spaide RF, Vitale AT, Toth IR, Oliver JM. Frosted branch angiitis associated with cytomegalovirus retinitis. Am $\mathcal{F}$ Ophthalmol 1992; 113: 522-8.

12 Keefe KS, Freeman WR, Peterson TJ, Wiley CA, Crapotta J, Quiceno JI, et al. Atypical healing of cytomegalovirus retinitis. Ophthalmology 1992; 99: 137784.

13 Jabs DA, Enger C, Haller J, de Bustros S. Retinal detachment in patients with cytomegalovirus retinitis. Arch Ophthalmol 1991; 109: 794-9.

14 Bachman DM, Bruni LM, DiGioia RA, Harris PJ, McMackin CM, Pistole MC. Visual field testing in the management of cytomegalovirus retinitis. Ophthalmology 1992; 99: 1393-9.

15 Holland GN, Buhles WC, Mastre B, Kaplan HJ. A controlled retrospective study of ganciclovir treatment for cytomegalovirus retinitis. Use of a standardised system for the assessment of disease outcome. Arch Ophthalmol 1989; 107: 1759-66.

16 Gass JDM. Inflammatory diseases of the retina and choroid. In: Stereoscopic atlas of macular diseases: diagnosis and treatment. St Louis: Mosby, 1987: 496-7.

17 Cochereau-Massin I, LeHoang P, Lautier-Frau M, Zerdoun E, Zazoun L, Robinet $\mathrm{M}$, et al. Ocular toxoplasmosis in human immunodeficiency virusinfected patients. Am F Ophthalmol 1992; 114: 130-5.

18 Holland GN, Engstrom RE, Glasgow BJ, Berger BB, Daniels SA, Sidikaro Y, et al. Ocular toxoplasmosis and AIDS. Am f Ophthalmol 1988; 106: 653-67.

19 Freeman WR, Wiley CA. In-situ nucleic acid hybridisation. Surv Ophthalmol $1989 ; 34: 187-92$.

20 Hennis HL, Scott AA, Apple DJ. Cytomegalovirus retinitis. Surv Ophthalmol 1989; 34: 193-203.

21 Palestine AG, Polis MA, de Smit MD, Baird BF, Fallon J, Kovacs JA, et al. A randomised, controlled trial of foscarnet in the treatment of cytomegalovirus retinitis in patients with AIDS. Ann Intern Med 1991; 115: 665-73.

22 Holland GN, Sidikaro Y, Kreiger AE. Treatment of cytomegalovirus retinitis with ganciclovir. Ophthalmology 1987; 94: 815-23.

23 Gross JG, Bouzette SA. Longitudinal study of cytomegalovirus retinitis in acquired immunodeficiency syndrome. Ophthalmology 1990; 97: 681-6.

24 Jabs DA, Newman C, de Bustros S, Polk BF. Treatment of cytomegalovirus retinitis. Ophthalmology 1987; 94: 824-30.

25 Jabs DA. Treatment of cytomegalovirus retinitis - 1992. [Editorial.] Arch Ophthalmol 1992; 110: 185-7.

26 Raviglione MC, Batten R, Pablos-Mendez A, Aceves-Casillas P, Mullen MP, Taranta A. Infections associated with Hickman catheters in patients with Taranta A. Infections associated with Hickman catheters in patient

27 Manischewitz JF, Quinnan GV Jr, Lane HC, Wittek AE. Synergistic effect of ganciclovir and foscarnet on cytomegalovirus replication in vitro. Antimicrob Agents Chemother 1990; 34: 373-5.

28 Nelson MR, Barter G, Hawkins D, Gazzard BG. Simultaneous treatment of cytomegalovirus retinitis with ganciclovir and foscarnet. Lancet 1991; 338: 250.

29 Ussery FM III, Gibson SR, Conklin RH, Piot DF, Stool EW, Conklin AJ. Intravitreal ganciclovir in the treatment of AIDS-associated cytomegalovirus retinitis. Ophthalmology 1989; 95: 640-8.

30 Henry K, Cantrill HL, Fletcher C, Chinnock BJ, Balfour HH. Use of intravitreal ganciclovir (dihydroxy propxy methyl guanine) for cytomegalovirus retinitis in a patient with AIDS. Am F Ophthalmol 1987; 103: 17-23.

31 Cantrill HL, Henry K, Melroe H, Knoblock WH, Ramsay RC, Balfour HH. Treatment of cytomegalovirus retinitis with intravitreal ganciclovir: long term results. Ophthalmology 1989; $96: 367-74$.

32 Heinemann MH. Long-term intravitreal ganciclovir for cytomegalovirus retinopathy. Arch Ophthalmol 1989; 107: 1767-72.

33 Diaz-Liopis M, Chipont E, Sanchez S, Espana E, Navea A, Menezo JL. Intravitreal foscarnet for cytomegalovirus retinitis in a patient with acquired immunodeficiency syndrome. Am f Ophthalmol 1992; 114: 742-7.

34 Sanborn GE, Anand R, Torti RE, Nightingale SD, Stanley XC, Yates B, et al. Sustained-release ganciclovir therapy for treatment, of cytomegalovirus retinitis. Arch Ophthalmol 1992; 110: 188-95

35 Sarraf D, Equi RA, Holland GN, Yoshizumi MO, Lee DA. Transscleral iontophoresis of foscarnet. Am F Ophthalmol 1993; 115: 748-54.

36 Egbert PR. Cytomegalovirus ocular infections. In: Darrell RW, ed. Viral diseases of the eye. Philadelphia: Lea \& Febiger, 1985.

37 Cochereau-Massin I, LeHoang P, Lautier-Frau M, Zazoun L, Marcel P, Robinet $M$, et al. Efficacy and tolerance of intravitreal ganciclovir in cytomegalovirus retinitis in acquired immune deficiency syndrome. Ophthalmology 1991; 98: 1348-55.

38 Polsky B, Wolitz R, Cantrill H, Chuang EL, Heinemann EH, Sands M, et al. Intravitreal ganciclovir salvage therapy for cytomegalovirus retinitis (ACTG Intravitreal ganciclovir salvage therapy for cytomegalovirus retinitis (ACTG 085): a preliminary report. In: Abstracts of
AIDS. Florence, Italy: 1991: 19-22 June.

39 Pepose JS, Holland GN, Nestor MS, Cochran AJ, Foos RY. Acquired immune deficiency syndrome. Pathogenic mechanisms of ocular disease. Ophthalmology 1985; 92: 472.

40 DeArmond B. Future directions in the management of cytomegalovirus infections. $\mathcal{F}$ Aids $1991 ; 4$ (suppl): S53-6.

41 Sidikaro Y, Silver L, Holland GN, Kreiger AE. Rhegmatogenous retinal detachment in patients with AIDS and necrotizing retinal infections. Ophthalmology 1991; 98: 129-35.

42 Holland GN. The management of retinal detachment in patients with acquired immunodeficiency syndrome. [Editorial] Arch Ophthalmol 1991; 109: 791-3.

43 Orellano J, Teich SA, Lieberman RM, Restrepo S, Peairs R. Treatment of retinal detachment in patients with acquired immune deficiency syndrome. Ophthalmology 1991; 98: 939-43.

44 Freeman WR, Quicenco JI, Crapotta JA, Listhaus A, Munguia D, Aguilar MF. Surgical repair of rhegmatogenous retinal detachment in immunosuppressed patients with cytomegalovirus retinitis. Ophthalmology 1992; 99: suppressed

45 Kuppermann BD, Petty JG, Richman DD, Mathews WC, Fullerton SC Rickman LS; et al. Correlation between CD4+ counts and prevalence of cytomegalovirus retinitis and human immunodeficiency virus-related noninfectious retinal vasculopathy in patients with acquired immunodeficiency syndrome. Am $\mathcal{F}$ Ophthalmol 1993; 115: 572-82. 